\title{
Elasto-plastic long-term behavior of granular soils: modelling and experimental validation
}

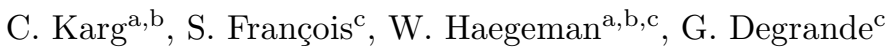 \\ ${ }^{a}$ Department of Industrial Engineering Sciences, KHBO, Zeedijk 101, B-8400 Ostend, Belgium \\ ${ }^{b}$ Department of Civil Engineering, Ghent University, Technologiepark 905, B-9052 Zwijnaarde, Belgium \\ ${ }^{c}$ Department of Civil Engineering, K.U.Leuven, Kasteelpark Arenberg 40, B-3001 Leuven, Belgium
}

\begin{abstract}
Repeated small amplitude dynamic loading of the soil in the vicinity of buildings, as arising from traffic or construction activities, may cause differential foundation settlements and structural damage. In this paper, an accumulation model for settlements due to vibrations at small strain levels in granular non-cohesive soils is proposed. It is assumed that the dynamic part of the stresses is small with respect to the static part. As plastic deformation in the soil is only observed after a considerable amount of dynamic loading cycles, only the accumulation of the average plastic deformation is considered. The model accounts for the dependency of the deformation on the stress state, the void ratio, and the dynamic loading amplitude. The model is calibrated for homogeneous fine grained sands with the aid of cyclic triaxial test results.
\end{abstract}

Keywords: Strain accumulation model, low level vibration, granular soil, long-term behavior

\section{Introduction}

Repeated dynamic loading of soils under low amplitude vibrations is a subject of growing interest $[1,6$, 9, 23]. Low amplitude ground vibrations are generated by road and railway traffic, subways, construction works (e.g. pile driving, tunneling or blasting) or heavy industry (e.g. machine foundations). These vibrations propagate through the soil and impinge on the foundations of nearby structures.

For small amplitude dynamic loading with resulting strain amplitudes below $10^{-4}$, no (measurable) residual deformation is observed for a single load cycle and the constitutive behavior of the soil can be described by linear visco-elastic models. After a large numbers of events, however, permanent deformation is observed in many cases $[9,18,23]$. The accumulation of strain is due to the rearrangement of grains, shear deformation, abrasion of soil particles, and non-recoverable drainage of pore water during cyclic loading. This phenomenon, characterized by elastic behavior on the short term and plastic behavior over a large number of load cycles, is referred to as strain accumulation [23]. On the long term, repeated dynamic loading of the soil in the vicinity of foundations may therefore result in a significant plastic deformation of the soil [5], causing differential foundation settlements and a considerable increase of the internal forces in the structure, resulting in structural damage.

A numerical model for the prediction of the accumulation of strain provides a proper understanding of the mechanical behavior of soil in the vicinity of foundations and allows for the prediction of foundation settlements. As the permanent deformations are only observed after a large number of load events, a constitutive model can be formulated that only describes the accumulation of the average permanent deformation per load cycle. This approach has originally been used to model metal fatigue $[7,10]$ and has also been adopted to compute settlements of roads $[1,2,8]$ and railway tracks $[19,20]$ under a large number of axle passages. This method is preferred above a step-by-step integration of the complete loading history as this

Email address: stijn.francois@bwk.kuleuven.be (S. François) 
would require a large computational time, even for problems of moderate size. A step-by-step integration of the entire loading history may also suffer from large numerical errors, as the accumulated strain per load cycle is of the same order of magnitude as the numerical error [14].

Suiker [19, 20] proposed a methodology for granular materials under large amplitude cyclic loading to assess deterioration of railway tracks. The long term constitutive behavior of ballast is based on a shakedown concept [16], where it is assumed that no permanent deformations occur if the cyclic load level is sufficiently small. If this elastic limit is exceeded, permanent deformations may gradually decrease or increase, which is referred to as shakedown and ratcheting, respectively. Suiker formulated the constitutive model in a form that shows strong analogies with the Perzyna visco-plastic model [15]. It is assumed that the permanent deformation is caused by two separate mechanisms, frictional sliding and volumetric compaction, which are accounted for through the use of two state variables in a classical plasticity framework.

The model of Suiker can only be rigorously applied if the cyclic loading amplitude can be computed as the quasi-static response of the subgrade due to the axle loads. However, the model makes no explicit distinction between the static and cyclic stress contributions, where the cyclic loading amplitude should be relatively large with respect to the static part. This assumption is admissible for the computation of the response of a railway track, but is not applicable underneath the foundations of structures where the cyclic part of the response is much smaller than the static pre-loading.

Niemunis et al. [14] formulated an accumulation model for granular materials under low amplitude cyclic loading, referred to as the Bochum accumulation model. In contrast to the model of Suiker, a tensorial formulation is used that aims to account for anisotropic effects during settlement such as a change of loading direction. The accumulation of deformation is non-vanishing and no shakedown behavior is encountered: compaction continues until a minimum void ratio is achieved. The model depends on a large number of parameters, for which an extensive amount of laboratory tests has been performed [23]. In order to obtain information on the anisotropic soil behavior, a torsional shear device and a cyclic multidimensional simple shear device have been used in addition to a cyclic triaxial test apparatus. Besides the need for a large number of model parameters, the incorporation of this model into a consistent finite element framework is not straightforward.

The aim of the present paper is to formulate an accumulation model for granular soils under low amplitude cyclic loading that is well suited for implementation in a finite element framework, where it is assumed that the cyclic part of the loading is small with respect to the static part, reflecting the stress conditions in the soil underneath a structure loaded by a low amplitude incident wave field. Some elements of the model of Suiker and the Bochum accumulation model are adopted.

The difference with Suiker's model is that explicit distinction is made between the static and cyclic part of the loading. The cyclic loading amplitude is regarded as a material parameter, while the equilibrium equation is solved for the average stress state. Furthermore, a different evolution law for the accumulated strains is formulated based on experimental observations. For the numerical integration, the sound framework of Suiker is applied to the new accumulation law.

This paper is organized as follows: in section 2, the general approach is outlined and the accumulation model is formulated. In section 3 , the accumulation model is calibrated for two medium coarse to coarse sands based on cyclic triaxial test results. In subsection 3.2, the model is calibrated in order to investigate the influence of the stress state on the accumulation behavior. The influence of the variation of the void ratio and the loading amplitude is discussed in subsection 3.3.

\section{Accumulation model}

\subsection{Strain decomposition}

A considerable amount of irrecoverable deformation is observed in soils after the application of a large number of low amplitude load cycles. An accumulation model describes the variation of the average permanent deformation per load cycle. The stresses and strains in a soil sample subjected to a low amplitude cyclic load are shown schematically in figure 1 . The stress tensor $\sigma_{i j}$ in the soil is decomposed as:

$$
\sigma_{i j}=\bar{\sigma}_{i j}+\sigma_{i j}^{\mathrm{cyc}}
$$


where $\bar{\sigma}_{i j}$ is the average (static) part of the stress tensor and $\sigma_{i j}^{\text {cyc }}$ is the small amplitude cyclic part of the stress tensor (figure 1). The mechanical sign convention is used where tensile stresses are positive. It is assumed that the cyclic part $\sigma_{i j}^{\text {cyc }}$ of the stress tensor is small with respect to the static part $\bar{\sigma}_{i j}$, reflecting the stress conditions in the soil underneath a structure loaded by a low amplitude incident wave field. As a result of the application of the cyclic stress, the soil deforms. The strain tensor $\epsilon_{i j}$ is similarly decomposed as:

$$
\epsilon_{i j}=\bar{\epsilon}_{i j}+\epsilon_{i j}^{\mathrm{cyc}}
$$

where $\bar{\epsilon}_{i j}$ is the average strain tensor and $\epsilon_{i j}^{\text {cyc }}$ is the cyclic part of the strain tensor (figure 1).

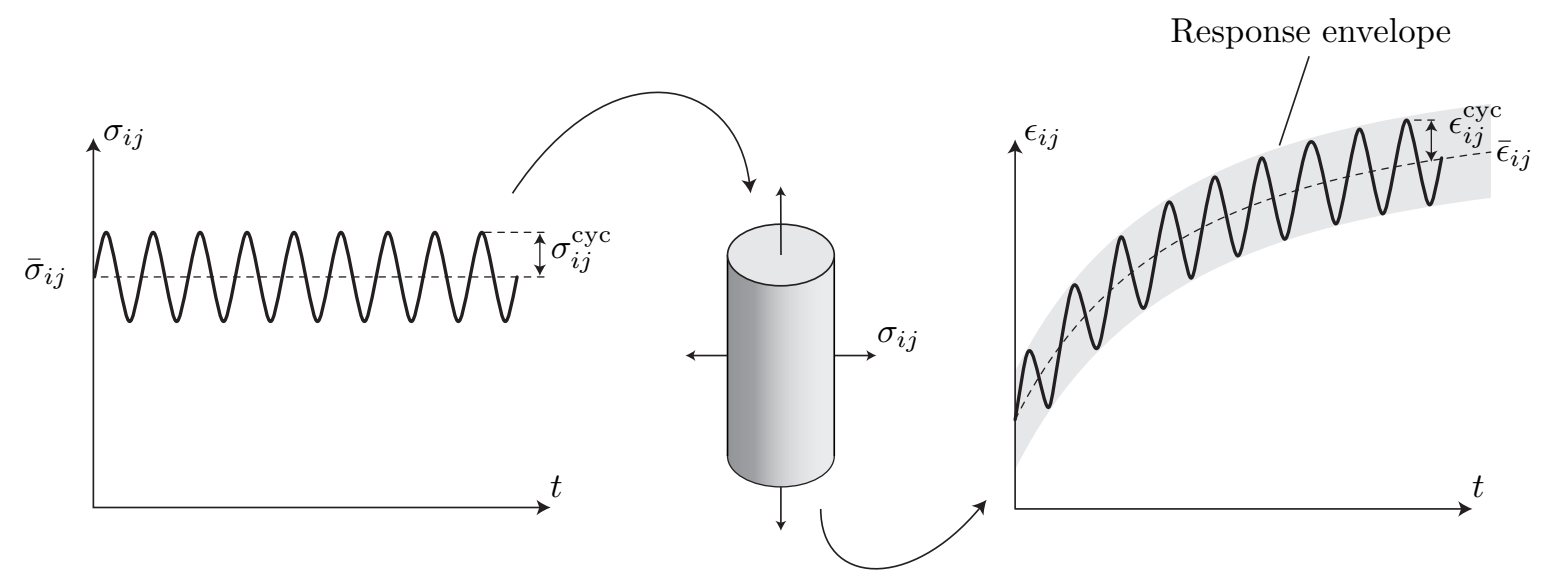

Figure 1: Long term and short term strain history $\epsilon_{i j}$ for soils under low amplitude cyclic loading $\sigma_{i j}$.

The average strain tensor $\bar{\epsilon}_{i j}$ gradually increases as the number of load cycles $N$ is increased. The average strain tensor $\bar{\epsilon}_{i j}$ is decomposed into a recoverable elastic part $\epsilon_{i j}^{\mathrm{e}}$ and an irrecoverable accumulated or plastic part $\epsilon_{i j}^{\text {acc }}$ as:

$$
\bar{\epsilon}_{i j}=\epsilon_{i j}^{\mathrm{e}}+\epsilon_{i j}^{\mathrm{acc}}
$$

The cyclic part $\epsilon_{i j}^{\text {cyc }}$ of the strain tensor varies on the short term as a direct result of the cyclic loading amplitude $\sigma_{i j}^{\text {cyc }}$. The cyclic part of the deformation $\epsilon_{i j}^{\text {cyc }}$ is assumed to be entirely recoverable and is governed by elastic material behavior.

In the following subsections, a new accumulation model is proposed for the accumulation of deformation. In subsection 2.2, the elastic material behavior for the recoverable part $\epsilon_{i j}^{\mathrm{e}}$ of the average strain and for the cyclic part of the strain $\epsilon_{i j}^{\text {cyc }}$ is discussed. Section 2.3 describes the irrecoverable part $\epsilon_{i j}^{\text {acc }}$ of the average strain, specified in terms of the accumulation rate $d \epsilon_{i j}^{\text {acc }} / d N$ with respect to the number of load cycles $N$.

\subsection{Elastic material behavior}

The elastic part of the deformation is determined by the constitutive relation:

$$
\bar{\sigma}_{i j}=C_{i j k l} \epsilon_{k l}^{\mathrm{e}}
$$

where $C_{i j k l}$ is the constitutive tensor:

$$
C_{i j k l}=\frac{3 K_{\mathrm{t}}}{2(1+\nu)}\left((1-2 \nu)\left(\delta_{i k} \delta_{j l}+\delta_{i l} \delta_{j k}\right)+2 \nu \delta_{i j} \delta_{k l}\right)
$$

defined in terms of the (constant) Poisson's ratio $\nu$, the tangent bulk modulus $K_{\mathrm{t}}$ and the Kronecker delta $\delta_{i j}$. In order to account for the increase in material stiffness for an increasing pressure, a pressure dependent elastic power law is adopted for the tangent bulk modulus [18, 22]:

$$
K_{\mathrm{t}}=K_{\mathrm{ref}}\left(\frac{p}{p_{\mathrm{ref}}}\right)^{1-n_{\mathrm{e}}}
$$


with $K_{\text {ref }}$ the reference bulk modulus, $p=\bar{\sigma}_{k k} / 3$ the hydrostatic pressure, $p_{\text {ref }}$ a reference pressure and $n_{\mathrm{e}}$ a model parameter. In combination with a constant Poisson's ratio $\nu$, this power law determines the elastic material behavior.

For the cyclic part $\epsilon_{i j}^{\text {cyc }}$ of the strain tensor, the same linear behavior is considered:

$$
\sigma_{i j}^{\mathrm{cyc}}=C_{i j k l} \epsilon_{k l}^{\mathrm{cyc}}
$$

Equation (7) is used in section 3 for the calibration of the bulk modulus $K_{\mathrm{t}}$.

\subsection{Accumulation of strain}

The accumulation of deformation is a result of particle rearrangements. Suiker [19, 20] distinguishes two distinct mechanisms: frictional sliding and volumetric compaction of soil particles. This assumption is adopted in the present model.

Volumetric compaction of soil particles is described by the accumulation of the volumetric strain tensor $\epsilon_{k k}^{\mathrm{acc}}$. Frictional sliding of soil particles is characterized by the deviatoric strain invariant $\kappa^{\text {acc }}$, defined as:

$$
\kappa^{\mathrm{acc}}=\sqrt{\frac{2}{3} e_{i j}^{\mathrm{acc}} e_{i j}^{\mathrm{acc}}}
$$

where $e_{i j}^{\mathrm{acc}}=\epsilon_{i j}^{\mathrm{acc}}-\epsilon_{k k}^{\mathrm{acc}} / 3 \delta_{i j}$ is the deviatoric strain.

As in classical plasticity, the material state is accounted for by the use of state variables. Two state variables $\kappa^{\text {acc,f }}$ and $\epsilon_{k k}^{\text {acc,c }}$ are introduced for frictional sliding and volumetric compaction, respectively. The accumulation rate $d \epsilon_{i j}^{\text {acc }} / d N$ is decomposed into a part generated by frictional sliding and a part generated by volumetric compaction:

$$
\frac{d \epsilon_{i j}^{\mathrm{acc}}}{d N}=\frac{d \kappa^{\mathrm{acc}}}{d N} m_{i j}^{\mathrm{f}}+\frac{d \epsilon_{k k}^{\mathrm{acc}, \mathrm{c}}}{d N} m_{i j}^{\mathrm{c}}
$$

where the unit tensors $m_{i j}^{\mathrm{f}}$ and $m_{i j}^{\mathrm{c}}$ represent the direction of accumulation for both mechanisms.

Besides a considerable deviatoric deformation, frictional sliding of soil particles results in a volumetric deformation of the soil as a result of dilation. The volumetric part of the accumulated strain $d \epsilon_{i j}^{\mathrm{acc}} / d N$ is related to both frictional sliding and volumetric compaction:

$$
\frac{d \epsilon_{k k}^{\mathrm{acc}}}{d N}=d_{\mathrm{f}} \frac{d \kappa^{\mathrm{acc}, \mathrm{f}}}{d N}+\frac{d \epsilon_{k k}^{\mathrm{acc}, \mathrm{c}}}{d N}
$$

where the parameter $d_{\mathrm{f}}$ controls the dilation induced by deviatoric deformation. The deviatoric part of the accumulated strain $d \epsilon_{i j}^{\text {acc }} / d N$ is entirely due to the frictional mechanism:

$$
\frac{d \kappa^{\mathrm{acc}}}{d N}=\frac{d \kappa^{\mathrm{acc}, \mathrm{f}}}{d N}
$$

Therefore, the superscript $\mathrm{f}$ will be omitted in the following.

\subsubsection{Direction of accumulation}

According to the experiments of Chang and Whitman [4] and Luong [11], the direction of accumulation only depends on the stress state. However, Wichtmann [23] detected a measurable influence of the number of load cycles on the direction of accumulation after large numbers of load cycles. This influence is neglected in the present model. Accordingly, the direction of accumulation is derived from the Mohr-Coulomb yield surface. This approach has also been adopted by Suiker [18] and is in correspondence with triaxial test results $[12,13,19,23]$. For frictional sliding the direction of plastic flow reads as:

$$
m_{i j}^{\mathrm{f}}=\frac{\partial q}{\partial \sigma_{i j}}+d_{\mathrm{f}} \frac{\partial p}{\partial \sigma_{i j}}=\frac{3}{2} \frac{s_{i j}}{q}+\frac{1}{3} d_{\mathrm{f}} \delta_{i j}
$$


where $s_{i j}=\sigma_{i j}-\sigma_{k k} / 3 \delta_{i j}$ is the deviatoric stress tensor and $q=\sqrt{3 / 2 s_{i j} s_{i j}}$ is the deviatoric stress invariant. For volumetric compaction, the deformation is purely volumetric:

$$
m_{i j}^{c}=\frac{\delta_{i j}}{3}
$$

Upon substitution of equations (12) and (13) into equation (9), the accumulated strain becomes:

$$
\frac{d \epsilon_{i j}^{\mathrm{acc}}}{d N}=\frac{d \kappa^{\mathrm{acc}}}{d N} \frac{3}{2} \frac{s_{i j}}{q}+\left(d_{\mathrm{f}} \frac{d \kappa^{\mathrm{acc}}}{d N}+\frac{d \epsilon_{k k}^{\mathrm{acc}, \mathrm{c}}}{d N}\right) \frac{\delta_{i j}}{3}
$$

\subsubsection{Accumulation rate}

The accumulation rates $d \kappa^{\mathrm{acc}} / d N$ and $d \epsilon_{k k}^{\mathrm{acc}, \mathrm{c}} / d N$ depend on a large number of parameters. Wichtmann [23] reviewed the effect of various parameters on strain accumulation and studied many dependencies experimentally. The parameters can be classified into three categories: the state of the soil, the stress state, and the characteristics of the cyclic loading. The soil is characterized by its void ratio, uniformity, grain size, granulometric composition, fabric (including intergranular structure, shape, surface roughness, mineralogy of grains), moisture content and historiotropy (including aging effects as cementation, abrasion, and static and cyclic preloading). The stress state is described by the hydrostatic stress $p$ and the stress ratio $\eta=q / p$. The dynamic loading is characterized by the number of load cycles, the amplitude and the cyclic loading frequency.

In order to account for the influence of these parameters, the assumption of a common compaction curve is made $[17,23]$. This implies that the shape of the compaction curve is invariant with respect to a variation of a parameter. Based on this assumption, a phenomenological law is put forward that describes the shape of the compaction curve. In cyclic triaxial tests, the compaction curve is characterized by an initial logarithmic growth of the deviatoric strain invariant as a function of the number of load cycles. After a certain number of load cycles, the growth becomes linear. Hence, the deviatoric accumulation $\kappa^{\text {acc }}$ is written in the form:

$$
\kappa^{\text {acc }}=c_{1} \ln \left(1+c_{2} N\right)+c_{3} N
$$

where $c_{1}, c_{2}$ and $c_{3}$ are constants. From equation (15), the deviatoric accumulation rate is found as:

$$
\frac{d \kappa^{\mathrm{acc}}}{d N}=\frac{c_{1} c_{2}}{1+c_{2} N}+c_{3}
$$

The deviatoric accumulation rate $d \kappa^{\text {acc }} / d N$ is subsequently formulated in terms of the state variable $\kappa^{\text {acc }}$, where the number of cycles $N$ is eliminated from equation (16). Therefore, it is assumed that the first term on the right hand side of equation (16) is only important at the onset of loading, during the initial logarithmic growth of the deformation. Neglecting the term $c_{3} N$ in the right hand side of equation (15) results in:

$$
\kappa^{\mathrm{acc}} \approx c_{1} \ln \left(1+c_{2} N\right)
$$

or equivalently:

$$
\frac{1}{1+c_{2} N} \approx \exp \left(-\frac{\kappa^{\mathrm{acc}}}{c_{1}}\right)
$$

Substition of equation (18) into equation (16) yields:

$$
\frac{d \kappa^{\mathrm{acc}}}{d N}=c_{1} c_{2} \exp \left(-\frac{\kappa^{\mathrm{acc}}}{c_{1}}\right)+c_{3}
$$

Equation (19) is written alternatively as:

$$
\frac{d \kappa^{\text {acc }}}{d N}=\alpha_{\mathrm{f}} \exp \left(-\theta_{\mathrm{f}} \kappa^{\text {acc }}\right)+\beta_{\mathrm{f}}
$$


where $\alpha_{\mathrm{f}}=c_{1} c_{2}$ and $\theta_{\mathrm{f}}=1 / c_{1}$ prescribe the initial exponential decrease of the accumulation rate and $\beta_{\mathrm{f}}=c_{3}$ corresponds to the final value of the accumulation rate after a large number of cycles. For the volumetric strains, a similar law is proposed:

$$
\frac{d \epsilon_{k k}^{\mathrm{acc}, \mathrm{c}}}{d N}=-\alpha_{\mathrm{c}} \exp \left(+\theta_{\mathrm{c}} \epsilon_{k k}^{\mathrm{acc}, \mathrm{c}}\right)-\beta_{\mathrm{c}}
$$

where $\alpha_{\mathrm{c}}, \theta_{\mathrm{c}}$ and $\beta_{\mathrm{c}}$ are model parameters. As the current model aims to predict settlements of soils under compressive stresses, the accumulated volumetric strain as well as the accumulation rate are negative. On the other hand, the deviatoric strain invariant and the deviatoric accumulation rate are positive. As positive model parameters are generally preferred, different signs are employed in equations (20) and (21).

The dependency of the model parameters $\alpha_{\mathrm{f}}, \theta_{\mathrm{f}}$ and $\beta_{\mathrm{f}}$ on the stress state $(p, q)$ is now further elaborated. The assumption of a common compaction curve $[17,23]$ implies that the ratio between two compaction curves at different stress ratios $\eta=q / p$ and $\eta_{0}=q_{0} / p_{0}$ is constant [23]:

$$
\kappa^{\mathrm{acc}}(N, \eta)=f_{\mathrm{f}}(\eta) \kappa^{\mathrm{acc}}\left(N, \eta_{0}\right)
$$

where $\left(p_{0}, q_{0}\right)$ is a reference stress state and $f_{\mathrm{f}}(\eta)$ is a scaling parameter. Equation $(22)$ is alternatively written as:

$$
\frac{d \kappa^{\mathrm{acc}}}{d N}(N, \eta)=f_{\mathrm{f}}(\eta) \frac{d \kappa^{\mathrm{acc}}}{d N}\left(N, \eta_{0}\right)
$$

Substituting equation (20) into equation (23) results in:

$$
\alpha_{\mathrm{f}}(\eta) \exp \left(-\theta_{\mathrm{f}}(\eta) \kappa^{\mathrm{acc}}(N, \eta)\right)+\beta_{\mathrm{f}}(\eta)=f_{\mathrm{f}}(\eta) \alpha_{\mathrm{f}}\left(\eta_{0}\right) \exp \left(-\theta_{\mathrm{f}}\left(\eta_{0}\right) \kappa^{\text {acc }}\left(N, \eta_{0}\right)\right)+f_{\mathrm{f}}(\eta) \beta_{\mathrm{f}}\left(\eta_{0}\right)
$$

Substituting equation (22) into equation (24) results in:

$$
\alpha_{\mathrm{f}}(\eta) \exp \left(-\theta_{\mathrm{f}}(\eta) \kappa^{\mathrm{acc}}(N, \eta)\right)+\beta_{\mathrm{f}}(\eta)=f_{\mathrm{f}}(\eta) \alpha_{\mathrm{f}}\left(\eta_{0}\right) \exp \left(-\theta_{\mathrm{f}}\left(\eta_{0}\right) \frac{\kappa^{\mathrm{acc}}(N, \eta)}{f_{\mathrm{f}}(\eta)}\right)+f_{\mathrm{f}}(\eta) \beta_{\mathrm{f}}\left(\eta_{0}\right)
$$

Equation (25) applies if:

$$
\begin{gathered}
\alpha_{\mathrm{f}}(\eta)=\alpha_{\mathrm{f}}^{0} f_{\mathrm{f}}(\eta) \\
\theta_{\mathrm{f}}(\eta)=\frac{\theta_{\mathrm{f}}^{0}}{f_{\mathrm{f}}(\eta)}
\end{gathered}
$$

and

$$
\beta_{\mathrm{f}}(\eta)=\beta_{\mathrm{f}}^{0} f_{\mathrm{f}}(\eta)
$$

where $\alpha_{\mathrm{f}}^{0}=\alpha_{\mathrm{f}}\left(\eta_{0}\right), \theta_{\mathrm{f}}^{0}=\theta_{\mathrm{f}}\left(\eta_{0}\right)$, and $\beta_{\mathrm{f}}^{0}=\beta_{\mathrm{f}}\left(\eta_{0}\right)$ are material parameters related to frictional sliding. The function $f_{\mathrm{f}}(\eta)$ accounts for the influence of the average stress state on the accumulation rate for frictional sliding. The following law is proposed in terms of the stress ratio $\eta=q / p$ :

$$
f_{\mathrm{f}}(\eta)=-\eta+\exp \left(-\left(\eta+\eta_{\mathrm{c}}\right)\right)
$$

where the critical stress ratio $\eta_{\mathrm{c}}$ equals $-6 \sin \phi_{\mathrm{c}} /\left(3-\sin \phi_{\mathrm{c}}\right)$ with $\phi_{\mathrm{c}}$ the critical friction angle. For stress ratios approaching the critical stress ratio $\eta_{\mathrm{c}}$, the function $f_{\mathrm{f}}(\eta)$ in equation $(29)$ increases exponentially, reflecting high accumulation rates for stress states close to the yield limit. The strain accumulation rate decreases with decreasing stress ratios. For isotropic stress conditions, i.e. $\eta=0, d \kappa^{\text {acc }} / d N$ vanishes as no deviatoric stresses are applied.

In a similar way, the dependency of the model parameters $\alpha_{\mathrm{c}}, \theta_{\mathrm{c}}$ and $\beta_{\mathrm{c}}$ on the stress state is elaborated based on the fact that the ratio of two compaction curves at different hydrostatic stresses $p$ and $p_{0}$ is constant:

$$
\epsilon^{\mathrm{acc}, \mathrm{c}}(N, p)=f_{\mathrm{c}}(p) \epsilon^{\mathrm{acc}, \mathrm{c}}\left(N, p_{0}\right)
$$


where $f_{\mathrm{c}}(\eta)$ is a scaling parameter. Equation (30) is alternatively written as:

$$
\frac{d \epsilon^{\mathrm{acc}, \mathrm{c}}}{d N}(N, p)=f_{\mathrm{c}}(\eta) \frac{d \epsilon^{\mathrm{acc}, \mathrm{c}}}{d N}\left(N, p_{0}\right)
$$

As for the deviatoric strains, this results in:

$$
\begin{gathered}
\alpha_{\mathrm{c}}(p)=\alpha_{\mathrm{c}}^{0} f_{\mathrm{c}}(p) \\
\theta_{\mathrm{c}}(p)=\frac{\theta_{\mathrm{c}}^{0}}{f_{\mathrm{c}}(p)}
\end{gathered}
$$

and

$$
\beta_{\mathrm{c}}(p)=\beta_{\mathrm{c}}^{0} f_{\mathrm{c}}(p)
$$

The parameters $\alpha_{\mathrm{c}}, \theta_{\mathrm{c}}$ and $\beta_{\mathrm{c}}$ only depend on the average effective mean pressure $p . \alpha_{\mathrm{c}}^{0}=\alpha_{\mathrm{c}}\left(p_{0}\right), \theta_{\mathrm{c}}^{0}=\theta_{\mathrm{c}}\left(p_{0}\right)$ and $\beta_{\mathrm{c}}^{0}=\beta_{\mathrm{c}}\left(p_{0}\right)$ are material parameters related to volumetric compaction.

The function $f_{\mathrm{c}}(p)$ accounts for the influence of the average effective mean pressure $p$ on the accumulation rate for volumetric compaction. For decreasing absolute values of hydrostatic pressures increasing accumulation rates are observed [23]. Therefore, the following law is proposed:

$$
f_{\mathrm{c}}(p)=\exp \left(C_{\mathrm{c}} p\right)
$$

where $C_{\mathrm{c}}$ is a model parameter. Equation (35) reaches its maximum 1 at $p=0$. For increasing compression, the hydrostatic pressure $p<0$ becomes more negative and $f_{\mathrm{c}}$ decreases.

\section{Model calibration}

\subsection{Accumulation model under triaxial conditions}

For the calibration of all model parameters, results of drained stress-controlled cyclic triaxial tests are used. Under triaxial conditions, the soil sample is confined by a hydrostatic stress $\sigma_{\mathrm{c}}$, while an additional vertical stress $\sigma_{\mathrm{v}}$ is applied. The stress state is axisymmetric around the vertical axis and is characterized by the hydrostatic stress $p=\sigma_{\mathrm{c}}+\frac{1}{3} \sigma_{\mathrm{v}}$ and the deviatoric stress invariant $q=\left|\sigma_{\mathrm{v}}\right|$ (figure 2). As a result of the application of these load cycles, a vertical deformation $\epsilon_{z z}$ and radial deformation $\epsilon_{r r}$ are observed. This deformation is characterized by the volumetric strain $\epsilon_{k k}=\epsilon_{z z}+2 \epsilon_{r r}$ and the deviatoric strain invariant $\kappa=\frac{2}{3}\left|\epsilon_{z z}-\epsilon_{r r}\right|$ that are energetically conjugate to the hydrostatic stress $p$ and the deviatoric stress invariant $q$, respectively.

In a cyclic triaxial test, the mean stress tensor is constant and the accumulation law can be integrated analytically. For the deviatoric strains, integration of equation (20) with respect to the number of cycles $N$ results in:

$$
\kappa^{\mathrm{acc}}=\frac{1}{\theta_{\mathrm{f}}} \ln \left(\exp \left(+\theta_{\mathrm{f}} \beta_{\mathrm{f}} N\right)\left(\frac{\alpha_{\mathrm{f}}}{\beta_{\mathrm{f}}}+\exp \left(+\theta_{\mathrm{f}} \kappa_{0}^{\text {acc }}\right)\right)-\frac{\alpha_{\mathrm{f}}}{\beta_{\mathrm{f}}}\right)
$$

Similarly, integration of equation (21) yields:

$$
\epsilon_{k k}^{\mathrm{acc}, \mathrm{c}}=-\frac{1}{\theta_{c}} \ln \left(\exp \left(+\theta_{\mathrm{c}} \beta_{\mathrm{c}} N\right)\left(\frac{\alpha_{\mathrm{c}}}{\beta_{\mathrm{c}}}+\exp \left(+\theta_{\mathrm{c}} \epsilon_{k k 0}^{\mathrm{acc}, \mathrm{c}}\right)\right)-\frac{\alpha_{\mathrm{c}}}{\beta_{\mathrm{c}}}\right)
$$

In equations (36) and (37), $\kappa_{0}^{\text {acc }}$ and $\epsilon_{k k 0}^{\text {acc,c }}$ denote the initial accumulated strain at the onset of cyclic loading, which can be assumed to be zero for virgin samples. The total accumulated volumetric strain equals:

$$
\epsilon_{k k}^{\mathrm{acc}}=d_{0} \kappa^{\mathrm{acc}}+\epsilon_{k k}^{\mathrm{acc}, \mathrm{c}}
$$




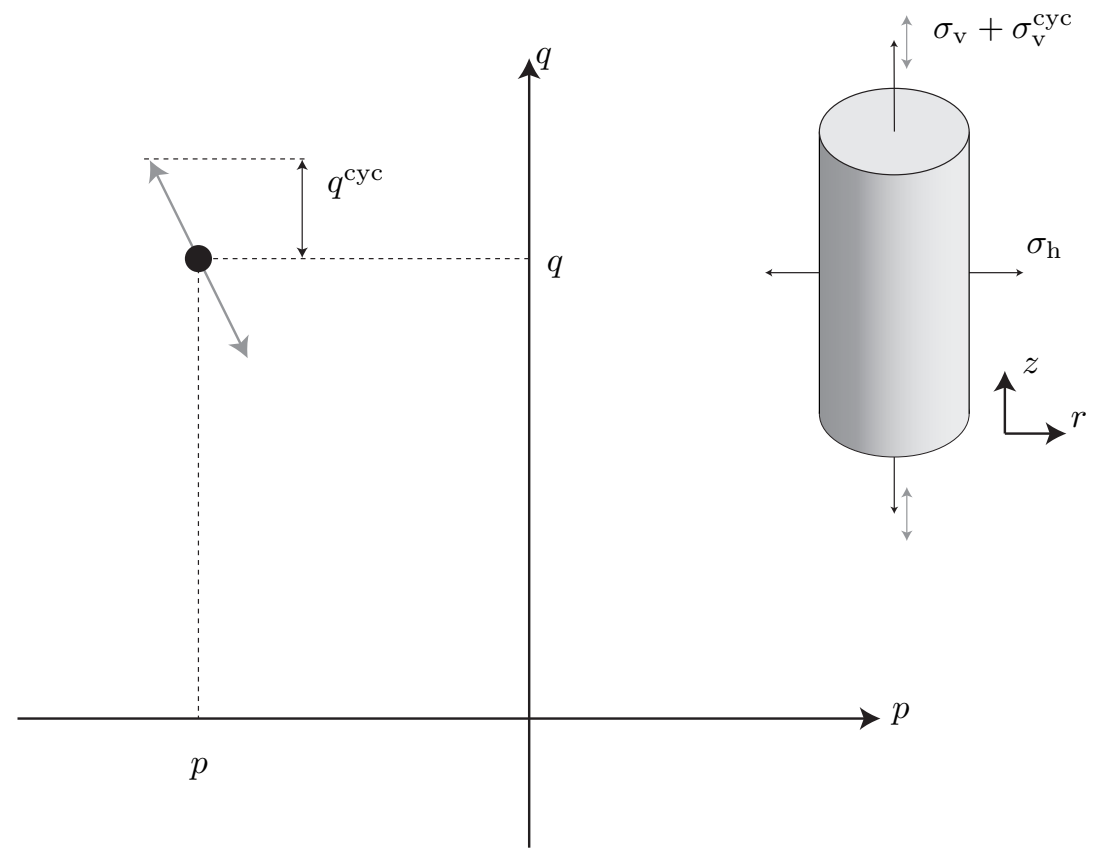

Figure 2: Stress state in a cyclic triaxial test.

\subsection{Model calibration with respect to the influence of stress conditions}

For the calibration of the accumulation model with respect to the influence of the stress state, cyclic triaxial test data from Wichtmann [23] are used. 20 cyclic triaxial tests at different stress levels $p$ and stress ratios $\eta$ are carried out, listed in Table 1 . The investigations are done on a uniform, medium coarse to coarse sand, referred to as sand No. 3 in the work of Wichtmann [23]. All samples are prepared with a relative density of about $60 \%$. The cyclic load ratio $\zeta=\sigma_{z z}^{\text {cyc }} / p$ is constant and equal to 0.30 in all tests. $N=100000$ load cycles are applied while the accumulated axial strain $\epsilon_{z z}^{\text {acc }}$ and accumulated volumetric strains $\epsilon_{k k}^{\text {acc }}$ are recorded. From these measurements $\kappa^{\text {acc }}$ and $\epsilon_{k k}^{\text {acc,c }}$ are computed as:

$$
\kappa^{\mathrm{acc}}=\frac{1}{3}\left|\epsilon_{k k}^{\mathrm{acc}}-\epsilon_{z z}^{\mathrm{acc}}\right|
$$

and

$$
\epsilon_{k k}^{\mathrm{acc}, \mathrm{c}}=d_{0} \kappa^{\mathrm{acc}}-\epsilon_{k k}^{\mathrm{acc}}
$$

\subsubsection{Elastic model parameters}

The elastic model parameters are derived from equation (7) that relates the cyclic stresses to the cyclic strains. Equation (7) is rewritten in order to relate the hydrostatic stress $p^{\text {cyc }}=1 / 3 \sigma_{k k}^{\text {cyc }}$ to the volumetric elastic strain $\epsilon_{k k}^{\mathrm{cyc}}$ as:

$$
p^{\mathrm{cyc}}=K_{\mathrm{t}} \epsilon_{k k}^{\mathrm{cyc}}
$$

In cyclic triaxial tests, the relation between $p^{\mathrm{cyc}}$ and $\epsilon_{k k}^{\mathrm{cyc}}$ is characterized by these hysteresis loops. Therefore, the elastic bulk modulus $K_{\mathrm{t}}$ for each sample is determined from the slope of the $\epsilon_{k k}^{\text {cyc }}-p^{\text {cyc }}$ hysteresis loops [9]. The hysteresis loop for the $20^{\text {th }}$ cycle is used, since it can be assumed that the sample has passed an initial conditioning. Furthermore, a constant Poisson's ratio $\nu=0.3$ is assumed, which is appropriate for medium dense compacted sands [3].

Next, the parameters $K_{\text {ref }}$ and $n_{\mathrm{e}}$ are determined by a linear least-squares regression, fitting equation (6) to the measurements on a double logarithmic scale. Selecting a reference pressure $p_{\text {ref }}=100 \mathrm{kPa}$, this results in $K_{\text {ref }}=133 \mathrm{MPa}$ and $n_{\mathrm{e}}=0.52$. Figure 3 shows a good correspondence between the predicted and measured bulk moduli. 


\begin{tabular}{|l|c|c|c|c|}
\hline & $\begin{array}{c}p \\
{[\mathrm{kPa}]}\end{array}$ & $\begin{array}{c}q \\
{[\mathrm{kPa}]}\end{array}$ & $\begin{array}{c}\eta \\
{[-]}\end{array}$ & $\begin{array}{c}\zeta \\
{[-]}\end{array}$ \\
\hline Test 1 & -100 & 25 & -0.250 & -0.300 \\
Test 2 & -100 & 50 & -0.500 & -0.300 \\
Test 3 & -100 & 100 & -1.000 & -0.300 \\
Test 4 & -100 & 125 & -1.250 & -0.300 \\
Test 5 & -100 & 131.3 & -1.313 & -0.300 \\
Test 6 & -200 & 75 & -0.375 & -0.300 \\
Test 7 & -200 & 100 & -0.500 & -0.300 \\
Test 8 & -200 & 125 & -0.625 & -0.300 \\
Test 9 & -200 & 150 & -0.750 & -0.300 \\
Test 10 & -200 & 175 & -0.875 & -0.300 \\
Test 11 & -200 & 200 & -1.000 & -0.300 \\
Test 12 & -200 & 225 & -1.125 & -0.300 \\
Test 13 & -200 & 237.6 & -1.188 & -0.300 \\
Test 14 & -200 & 262.6 & -1.313 & -0.300 \\
Test 15 & -200 & 275 & -1.375 & -0.300 \\
Test 16 & -300 & 75 & -0.250 & -0.300 \\
Test 17 & -300 & 150 & -0.500 & -0.300 \\
Test 18 & -300 & 300 & -1.000 & -0.300 \\
Test 19 & -300 & 375 & -1.250 & -0.300 \\
Test 20 & -300 & 393.3 & -1.313 & -0.300 \\
\hline
\end{tabular}

Table 1: Stress conditions for the tests of Wichtmann [23] used for model calibration.

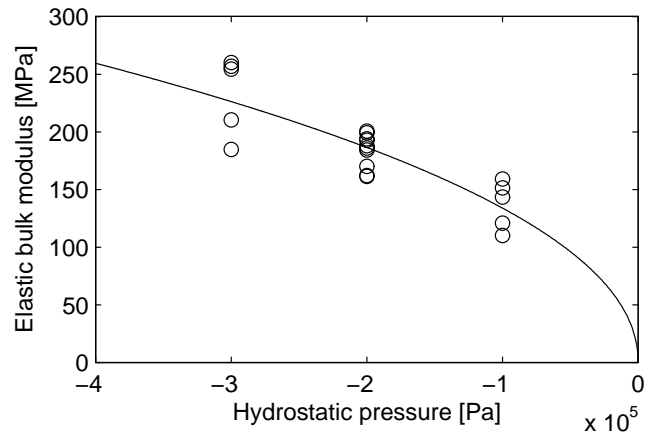

Figure 3: Elastic bulk modulus $K$ as a function of the hydrostatic pressure $p$. The predicted bulk modulus (solid line) and the experimental bulk modulus (circles) are compared. 


\subsubsection{Accumulation model parameters}

A non-linear least-squares procedure is used to fit curves (36) to the test data for the accumulated deviatoric strain. This procedure results in the parameters $\alpha_{\mathrm{f}}^{0}=2.45 \times 10^{-4}, \beta_{\mathrm{f}}^{0}=2.30 \times 10^{-8}, \theta_{\mathrm{f}}^{0}=1162$, $C_{\mathrm{f}}=5.73$, and $\eta_{\mathrm{c}}=-1.30$. The calibrated parameter $\eta_{\mathrm{c}}=-1.30$ corresponds to the actual critical stress ratio $\eta \approx-1.25$ of the sand under consideration. Figure 4 compares the experimental data to the respective calibrated accumulation curve, where a good correspondence is observed.

Subsequently, a non-linear least squares procedure is used to fit the curves (37) to the test data for the accumulated volumetric strain. This procedure results in $\alpha_{\mathrm{c}}^{0}=1.12 \times 10^{-4}, \beta_{\mathrm{c}}^{0}=4.42 \times 10^{-8}, \theta_{\mathrm{c}}^{0}=1367$, $C_{\mathrm{c}}=3.30 \times 10^{-7} \mathrm{~Pa}^{-1}$, and $d_{0}=0.31$. The calibrated dilation $d_{0}=0.31$ is in correspondence with values found in literature ranging from $d_{0}=0.20$ to $d_{0}=0.35$ [18]. Figure 5 compares the experimental data with the respective calibrated accumulation curve.

In order to further assess the modeling error, the accumulated strains $\epsilon_{r r}^{\mathrm{acc}}$ and $\epsilon_{z z}^{\mathrm{acc}}$ are computed for each test from equation (14). Figures 6 and 7 compare the recorded data of tests 1 to 20 to the respective predicted curves.

Good agreement between model and test data is achieved for all tests and at different hydrostatic stresses $p$. This demonstrates that the present model is suitable for the description of accumulation behavior of granular soil.

\subsubsection{Prediction of the dilative behavior at large stress ratios}

While the $p$-dependence of $\epsilon_{k k}^{\text {acc }}$ is directly described by the function $f_{c}(p)$, the $\eta$-dependence of $\epsilon_{k k}^{\mathrm{acc}}$ is due to the portion $d_{0}$ of $\kappa^{\text {acc }}$. This dependence is studied by computing the accumulated volumetric strain from equation (14):

$$
\frac{d \epsilon_{k k}^{\mathrm{acc}}}{d N}=d_{0} \frac{d \kappa^{\mathrm{acc}}}{d N}+\frac{d \epsilon_{k k}^{\mathrm{acc}, c}}{d N}
$$

Introducing equations (20) and (21) yields:

$$
\frac{d \epsilon_{k k}^{\mathrm{acc}}}{d N}=d_{0}\left[\alpha_{\mathrm{f}} \exp \left(-\theta_{\mathrm{f}} \kappa^{\mathrm{acc}}\right)+\beta_{\mathrm{f}}\right]-\left[\alpha_{\mathrm{c}} \exp \left(+\theta_{\mathrm{c}} \epsilon_{k k}^{\mathrm{acc}, \mathrm{c}}\right)+\beta_{\mathrm{c}}\right]
$$

At the onset of loading, where $\kappa^{\text {acc }}=0$ and $\epsilon_{k k}^{\text {acc,c }}=0$, the accumulated volumetric strain rate equals:

$$
\frac{d \epsilon_{k k}^{\mathrm{acc}}}{d N}=d_{0}\left(\alpha_{\mathrm{f}}^{0}+\beta_{\mathrm{f}}^{0}\right) f_{\mathrm{f}}(\eta)-\left(\alpha_{\mathrm{c}}^{0}+\beta_{\mathrm{c}}^{0}\right) f_{\mathrm{c}}(p)
$$

The accumulated volumetric strain rate is plotted in figure 8 as a function of the stress state. This demonstrates that the model correctly predicts a change from compactive behavior at low stress ratios $\eta$ to a dilative behavior near the critical state line.

\subsection{Model calibration with respect to the influence of void ratio and cyclic strain amplitude}

In this section, the presented accumulation model is applied for the prediction of accumulation under varying void ratio and cyclic strain amplitude. The model is calibrated using a number of triaxial tests on sand of Mol [9], a uniform fine grained sand comparable to the sand No. 3 used in the tests of Wichtmann [23]. The samples were prepared using moist tamping with undercompaction. This technique allows for the preparation of samples with target densities and results in a sufficiently homogeneous samples and test repeatability [21]. The samples are subjected to drained stress-controlled tests [9].

Six tests have been performed at a constant stress state $(p=-200 \mathrm{kPa}, \eta=-0.75)$ but with varying void ratio and cyclic loading amplitude (table 2). The void ratio ranges from $e=0.691$ to $e=0.8$ while the cyclic loading amplitude, defined as $\epsilon^{\mathrm{amp}}=\sqrt{\left(\epsilon_{r r}^{\mathrm{cyc}}\right)^{2}+2\left(\epsilon_{z z}^{\mathrm{cyc}}\right)^{2}}$, ranges from 0.0085 to 0.0245 . 


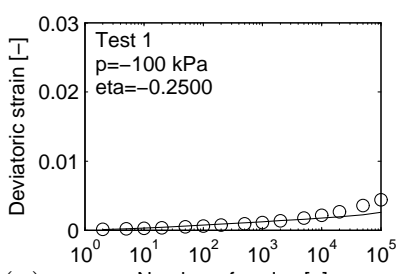

(a)

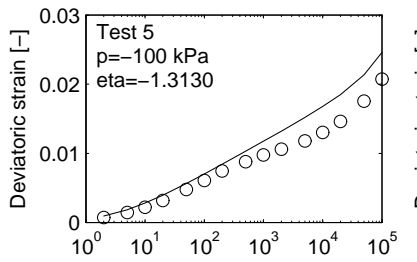

(e)

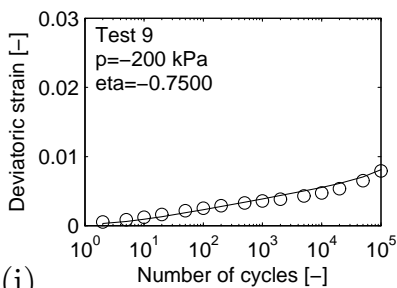

(i)

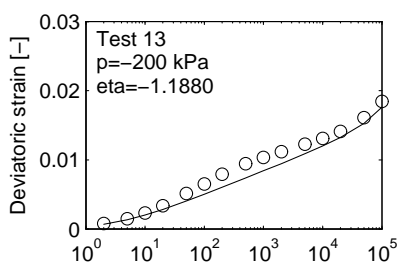

(m)

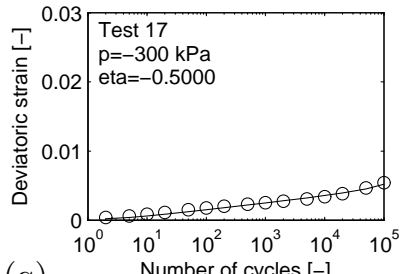

(q) Number of cycles [-]

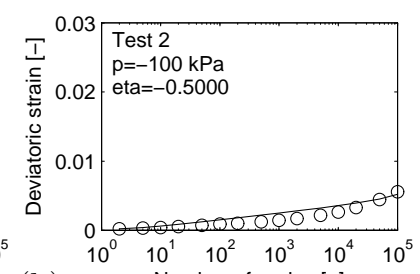

(b)

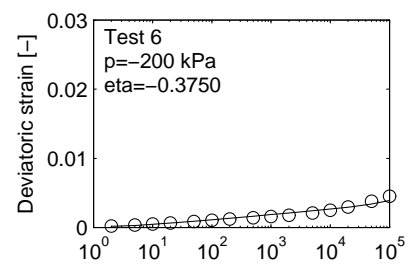

(f)

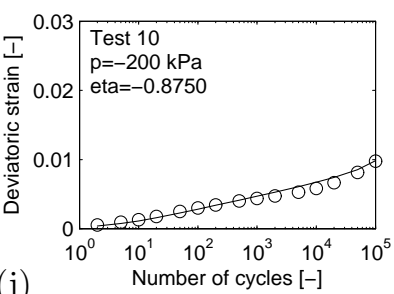

(j)

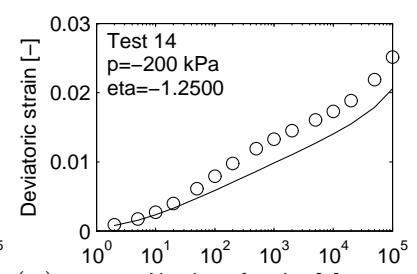

(n)

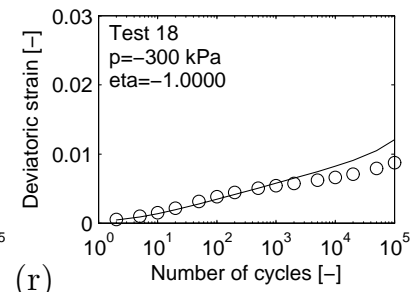

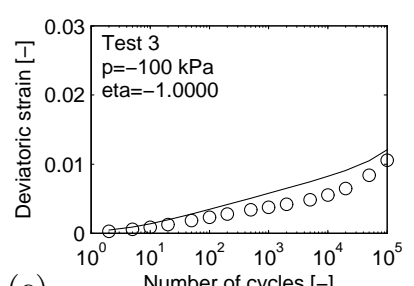

(c)

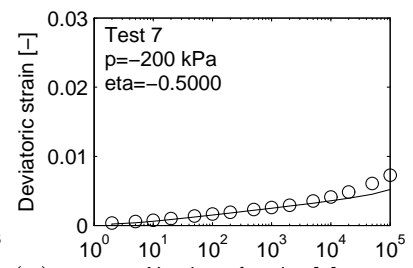

(g)

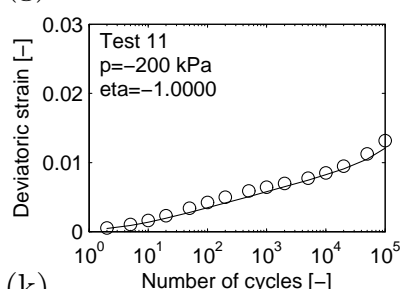

(k)

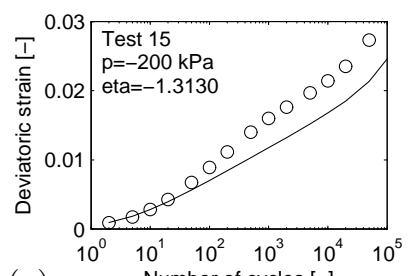

(o)

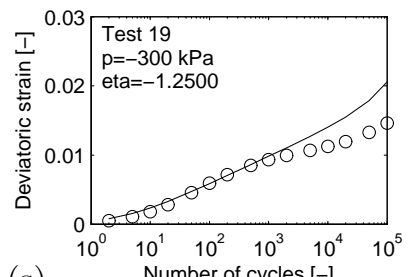

(s)

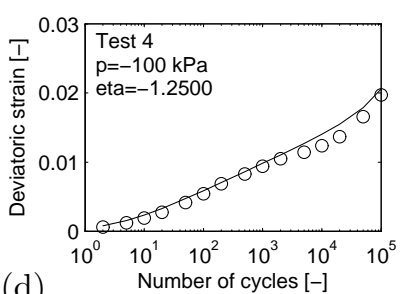

(d)

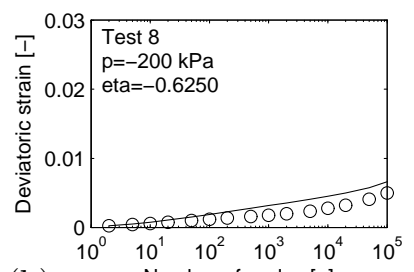

(h)

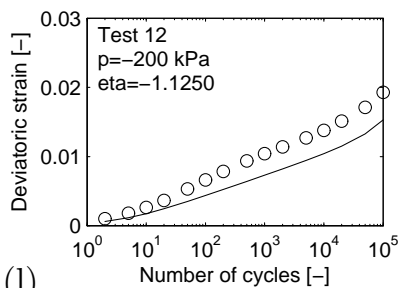

(1)

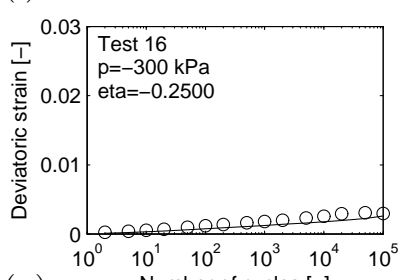

(p)

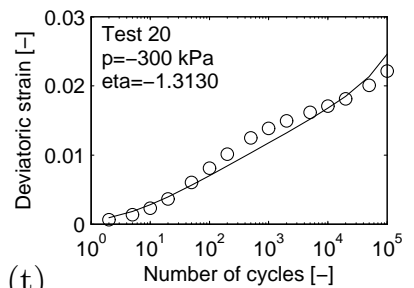

Figure 4: Measured and calibrated accumulated deviatoric strains $\kappa^{\text {acc }}$ for tests of Wichtmann [23]. 


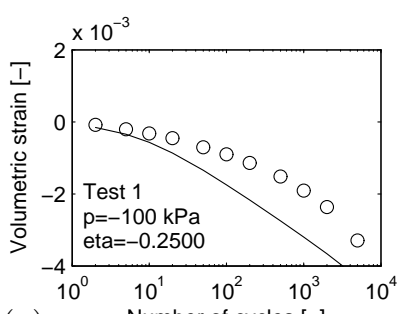

(a)

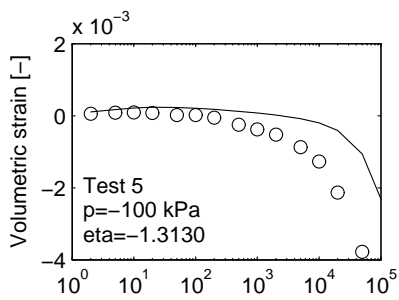

(e)

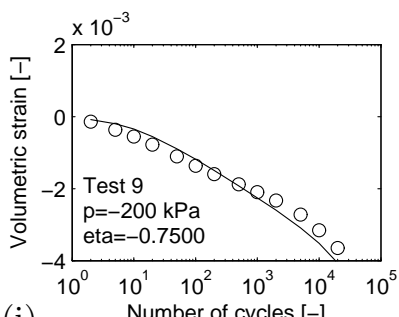

(i)

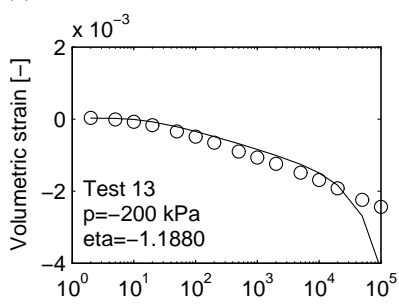

(m)

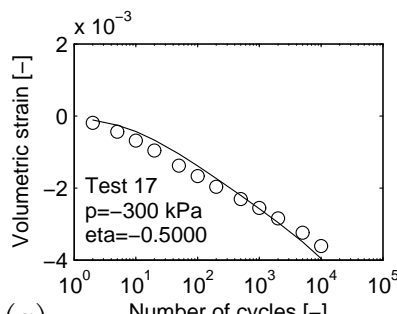

(q)

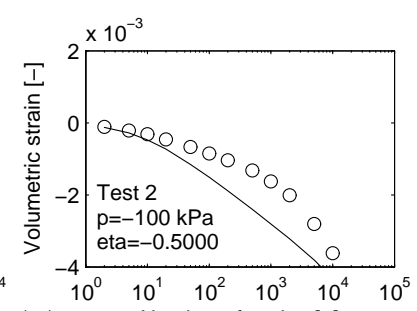

(b)

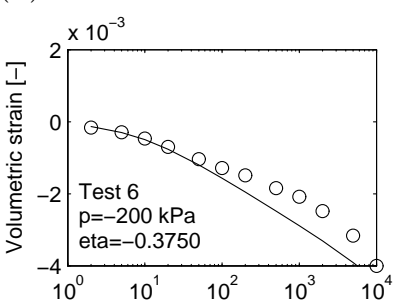

(f)

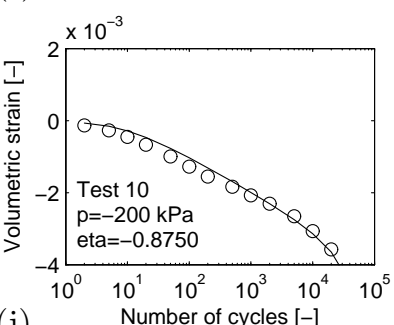

(j)

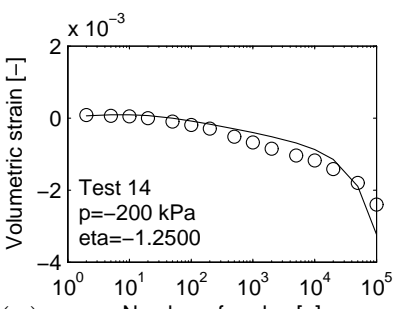

(n)

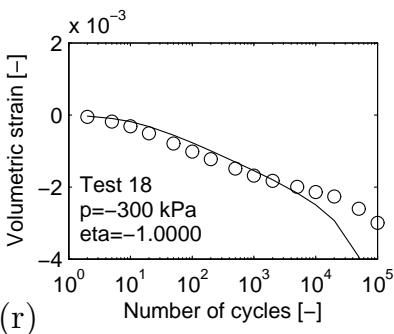

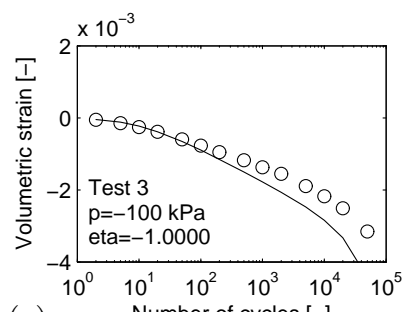

(c)

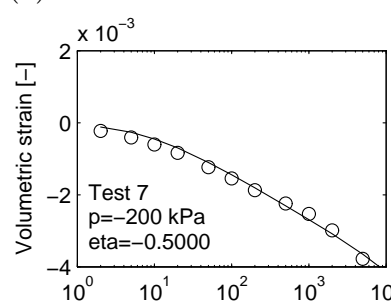

(g)

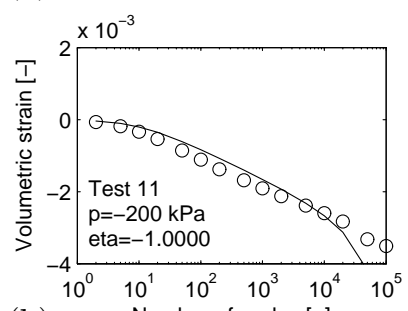

(k)

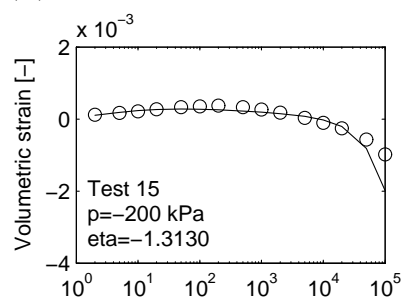

(o)

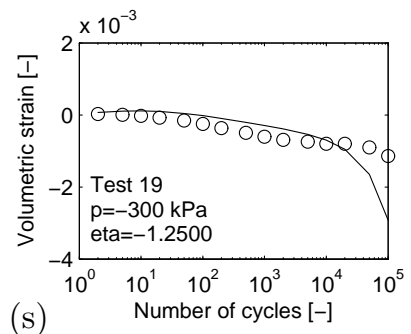

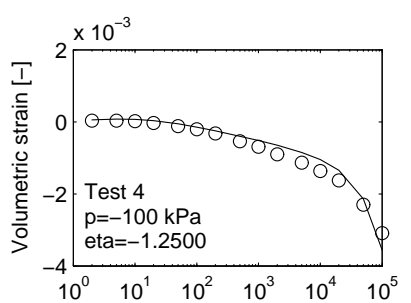

(d)

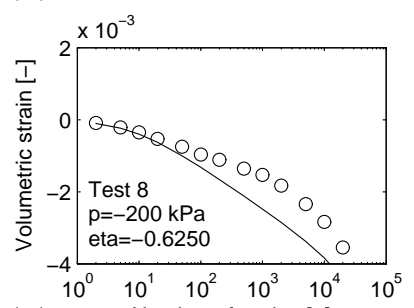

(h)

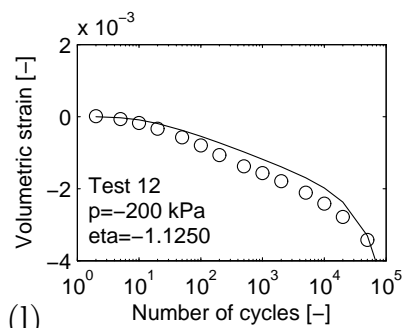

(1)

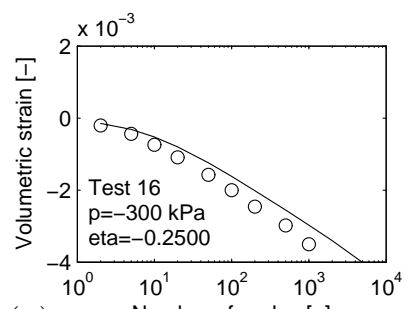

(p)

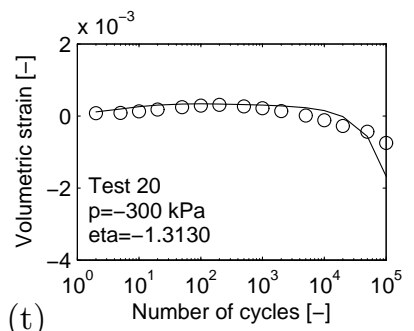

Figure 5: Measured and calibrated deviatoric volumetric strains $\epsilon_{k k}^{\mathrm{acc}}$ for tests of Wichtmann [23]. 


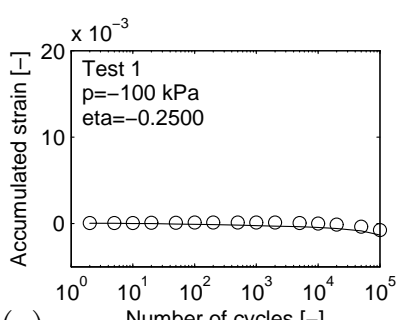

(a) Number of cycles [-] (b)

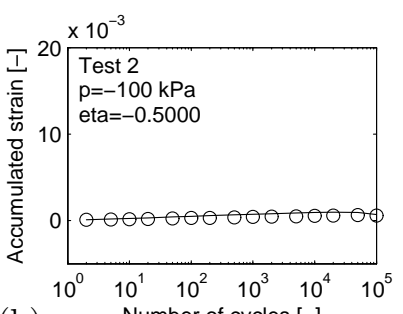

(b)

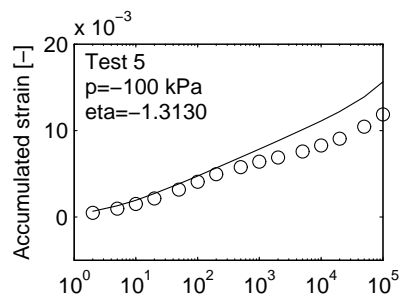

(e) Number of cycles [-]

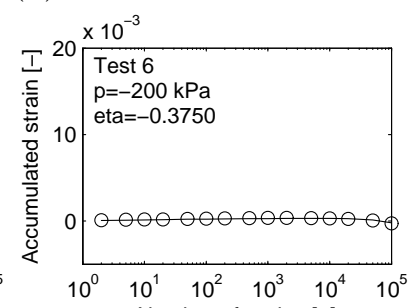

(f)
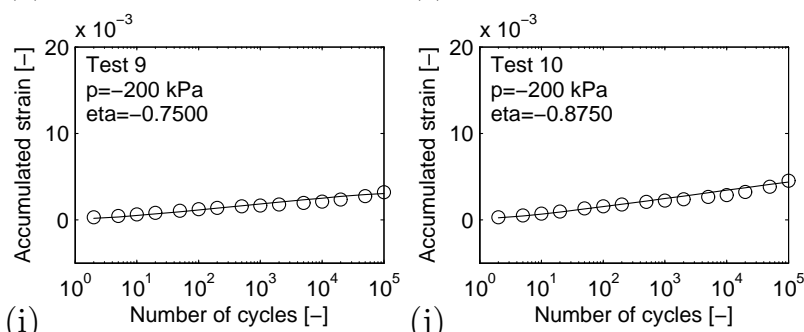

(j)

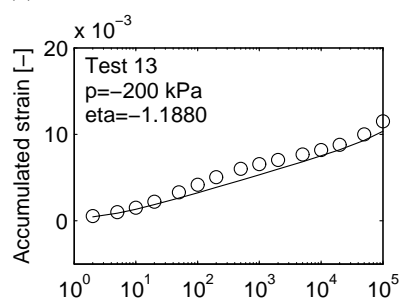

(m)

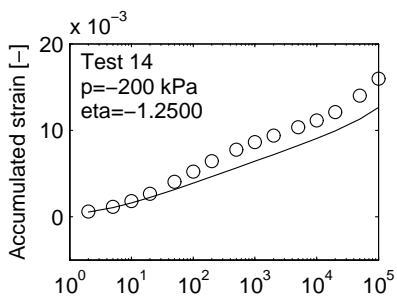

(n) Number of cycles [-]

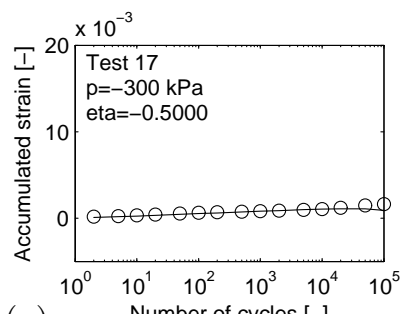

(q) Number of cycles [-]

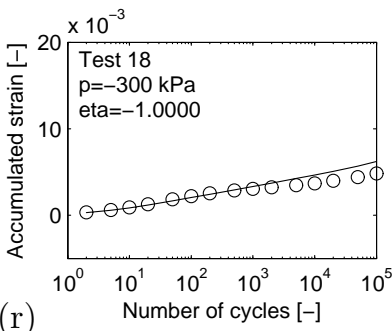

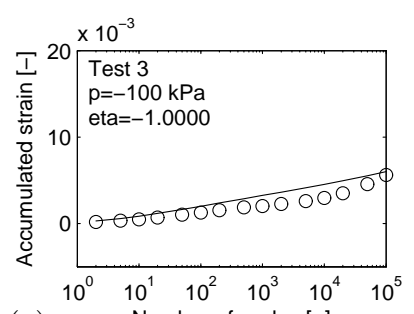

(c) Number of cycles [-]

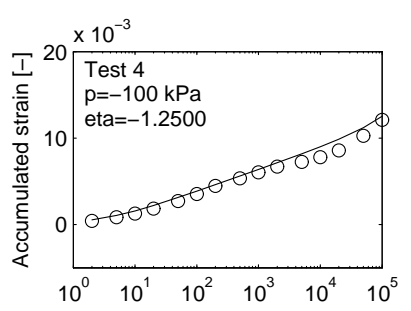

(d)

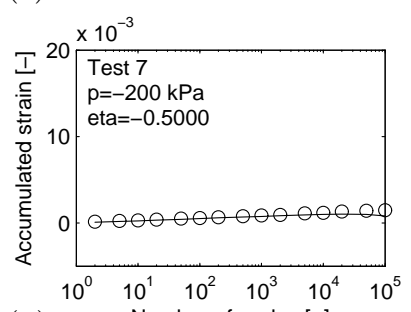

(g)

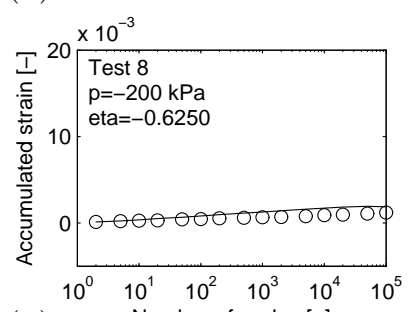

(h) Number of cycles [-]
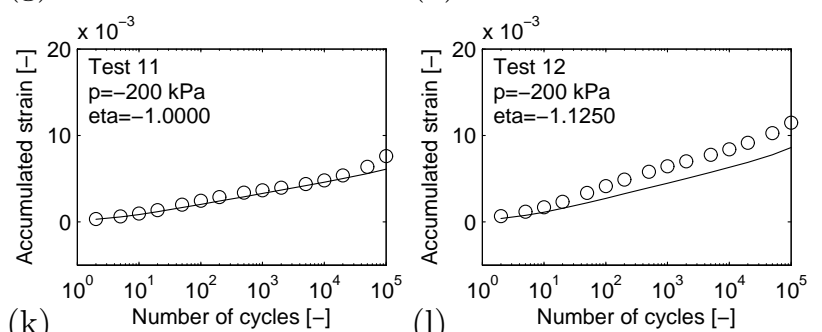

(k) Number of cycles [-] (l)

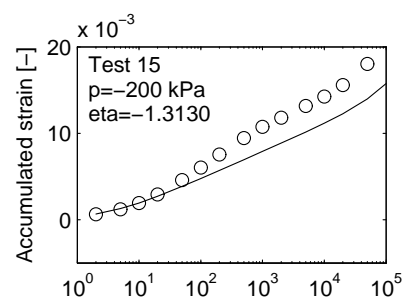

(o)
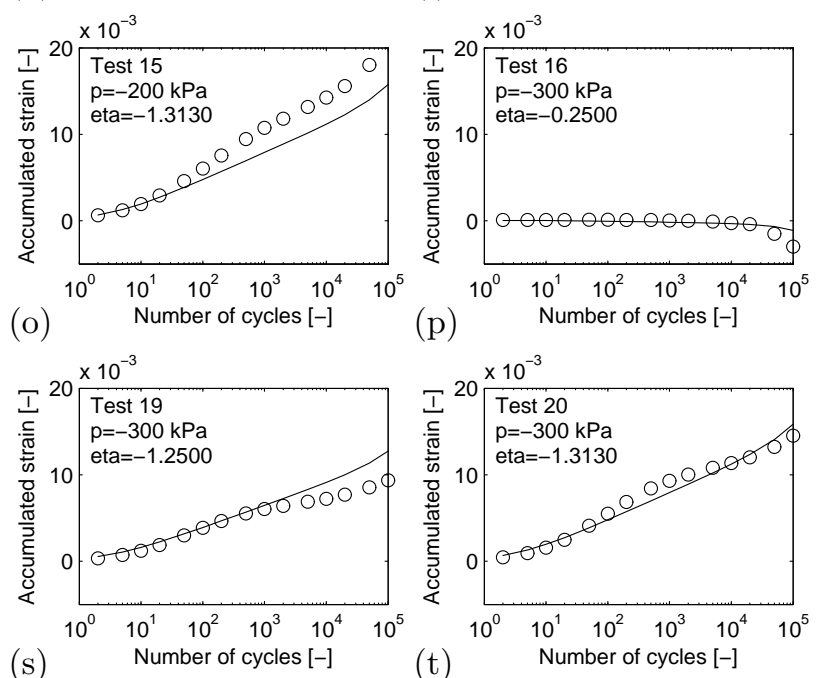

(p)

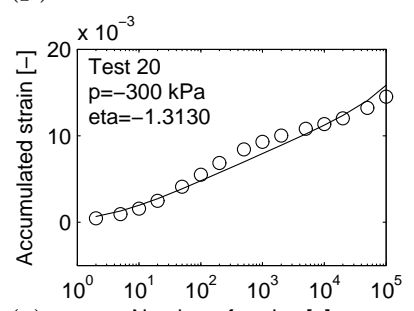

(t)

Figure 6: Measured and calibrated strains $\epsilon_{1}^{\text {acc }}$ for tests of Wichtmann [23]. 

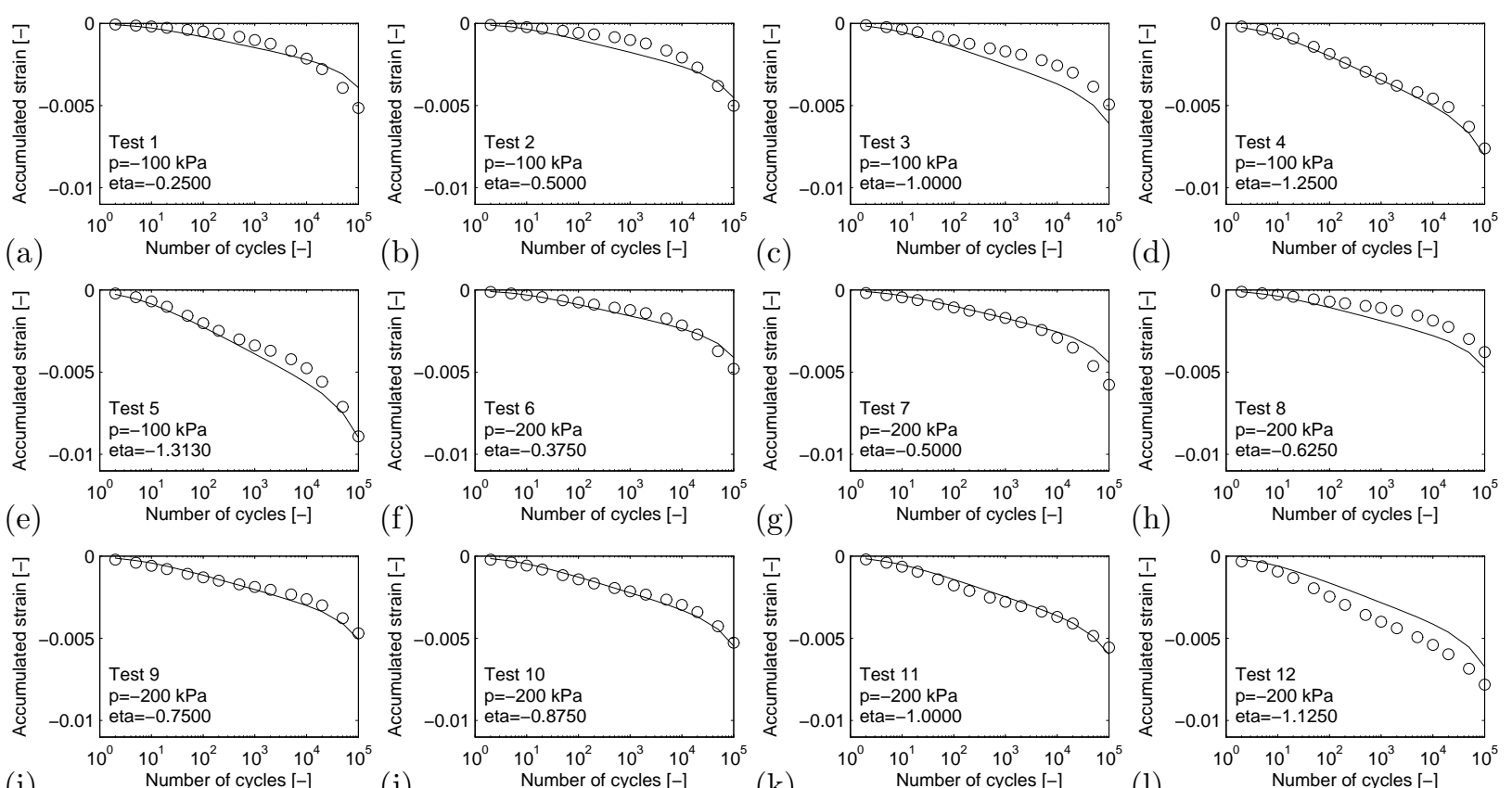

(g)
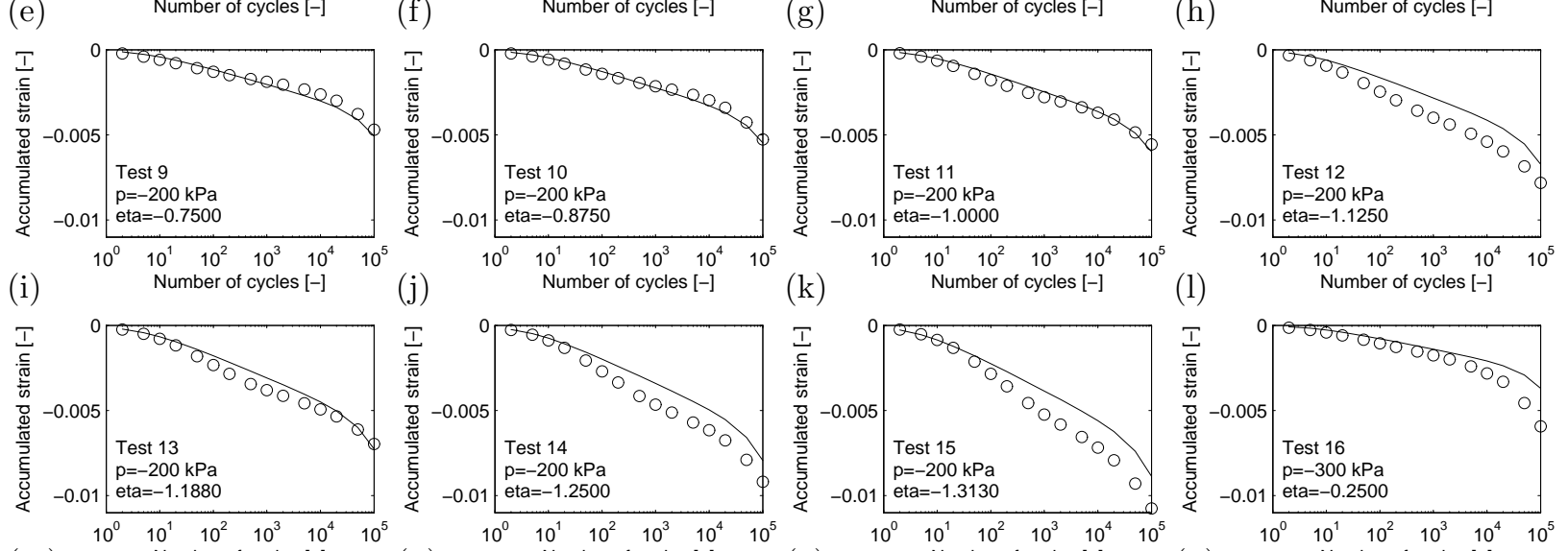

(n) Number of cycles [-] (o)
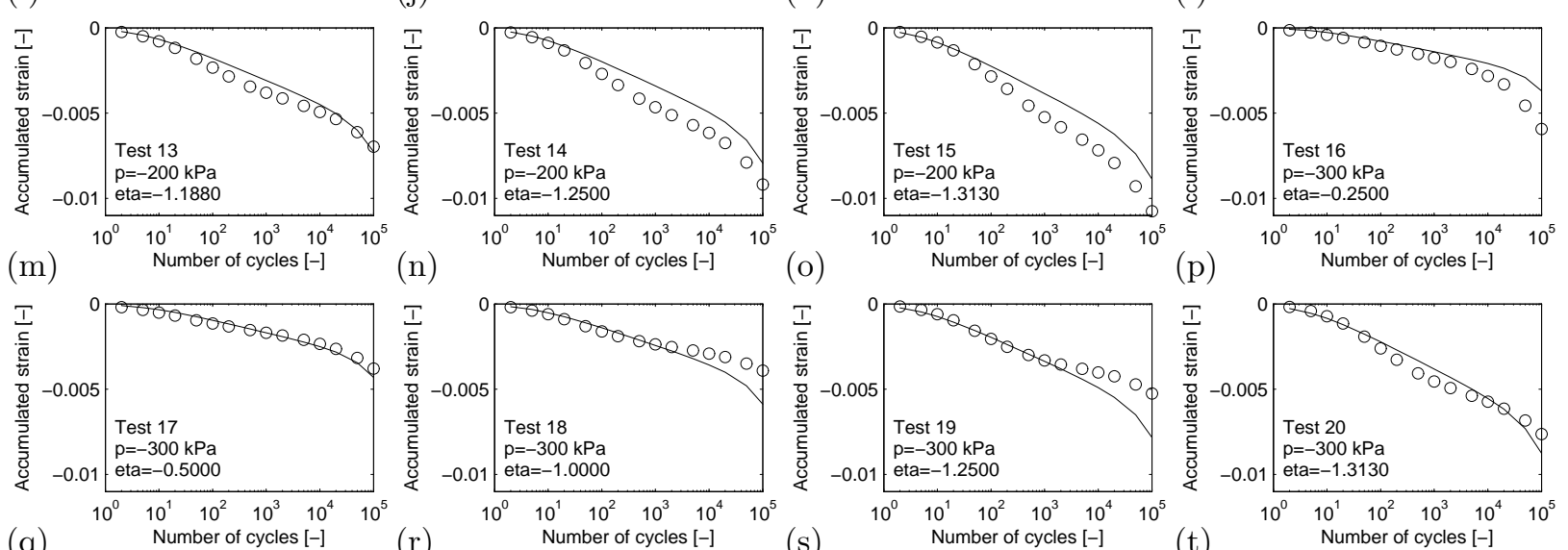

Figure 7: Measured and calibrated strains $\epsilon_{3}^{\text {acc }}$ for tests of Wichtmann [23].

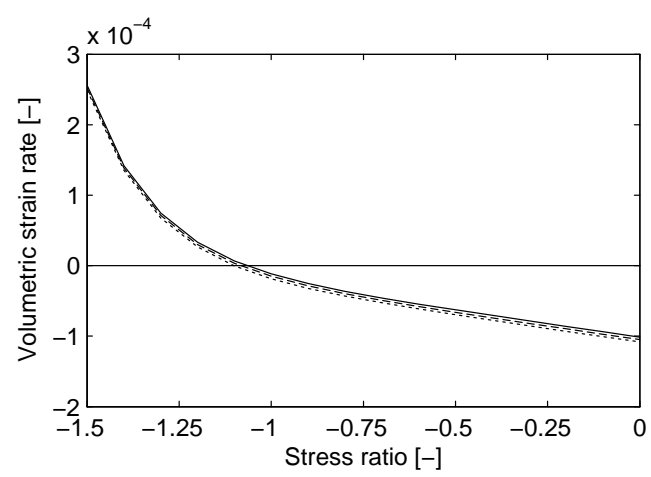

Figure 8: Accumulated volumetric strain rate $d \epsilon_{k k}^{\mathrm{acc}} / d N$ at the onset of loading $\left(\kappa^{\mathrm{acc}}=0\right.$ and $\left.\epsilon_{k k}^{\text {acc,c }}=0\right)$ as a function of the stress ratio $\eta$ at $p=-100 \mathrm{kPa}$ (solid line), $p=-200 \mathrm{kPa}$ (dashed line), and $p=-300 \mathrm{kPa}$ (dotted line). 


\begin{tabular}{|c|c|c|c|c|c|}
\hline Test & $\begin{array}{c}e \\
{[-]}\end{array}$ & $\begin{array}{c}\epsilon_{z z}^{\text {cyc }} \\
{[\%]}\end{array}$ & $\begin{array}{c}p \\
{[\mathrm{kPa}]}\end{array}$ & $\begin{array}{c}\eta \\
{[\mathrm{kPa}]}\end{array}$ & $\begin{array}{c}K_{\text {ref }} \\
{[\mathrm{MPa}]}\end{array}$ \\
\hline LT1 & 0.800 & 0.0135 & -200 & -0.75 & 120 \\
LT2 & 0.775 & 0.0130 & -200 & -0.75 & 120 \\
LT3 & 0.691 & 0.0085 & -200 & -0.75 & 180 \\
LT4 & 0.740 & 0.0215 & -200 & -0.75 & 120 \\
LT5 & 0.709 & 0.0245 & -200 & -0.75 & 130 \\
LT6 & 0.745 & 0.0230 & -200 & -0.75 & 130 \\
\hline
\end{tabular}

Table 2: Reference bulk moduli $K_{\text {ref }}$ for different long term accumulation tests on the sand of Mol.

\subsubsection{Elastic model parameters}

The elastic material properties of the sand of Mol are similar to the properties of sand No. 3. Accordingly, parameters $\nu, p_{\text {ref }}$, and $n_{\mathrm{e}}$, calibrated for sand No. 3 are adopted here for the sand of Mol. The tangent bulk modulus $K_{\mathrm{t}}$ differs with varying strain amplitudes and void ratios. Therefore, the reference bulk modulus $K_{\text {ref }}$ is determined for each test separately. Table 2 lists the corresponding values together with the respective void ratio $e$ after consolidation and strain amplitude $\epsilon_{z z}^{\text {cyc }}$.

\subsubsection{Accumulation model parameters}

In order to account for the influence of the void ratio and the loading amplitude, Equations (26) to (28) are modified as:

$$
\begin{aligned}
\alpha_{\mathrm{f}}(\eta) & =\alpha_{\mathrm{f}}^{0} f_{\mathrm{f}} f_{e} f_{\mathrm{ampl}} \\
\theta_{\mathrm{f}}(\eta) & =\frac{\theta_{\mathrm{f}}^{0}}{f_{\mathrm{f}} f_{e} f_{\mathrm{ampl}}} \\
\beta_{\mathrm{f}}(\eta) & =\beta_{\mathrm{f}}^{0} f_{\mathrm{f}} f_{e} f_{\mathrm{ampl}}
\end{aligned}
$$

Similarly, equations (32) to (34) are modified as:

$$
\begin{aligned}
\alpha_{\mathrm{c}}(p) & =\alpha_{\mathrm{c}}^{0} f_{\mathrm{c}} f_{e} f_{\mathrm{ampl}} \\
\theta_{\mathrm{c}}(p) & =\frac{\theta_{\mathrm{c}}^{0}}{f_{\mathrm{c}} f_{e} f_{\mathrm{ampl}}} \\
\beta_{\mathrm{c}}(p) & =\beta_{\mathrm{c}}^{0} f_{\mathrm{c}} f_{e} f_{\mathrm{ampl}}
\end{aligned}
$$

where $f_{e}$ and $f_{\text {ampl }}$ account for the dependency on the void ratio $e$ and the cyclic loading amplitude, respectively. Due to the limited amount of test data, no new formulation is proposed and the dependencies are adopted from Wichtmann [23]:

$$
f_{e}=\frac{\left(C_{e}-e\right)^{2}}{\left(C_{e}-e_{\mathrm{ref}}\right)^{2}} \frac{1+e_{\mathrm{ref}}}{1+e}
$$

Equation (51) accounts for the dependency on the void ratio $e$ by means of two model parameters $C_{e}$ and $e_{\text {ref. }}$.

The function $f_{f}$ is defined as:

$$
f_{f}=\left(\frac{\epsilon^{\mathrm{amp}}}{\epsilon_{\mathrm{ref}}^{\mathrm{amp}}}\right)^{2}
$$

and accounts for the dependency on the cyclic loading amplitude $\epsilon^{\mathrm{amp}}$ where $\epsilon_{\mathrm{ref}}^{\mathrm{amp}}$ is a reference amplitude. For the present experiment, $\epsilon_{\mathrm{ref}}^{\mathrm{amp}}=0.003$ is considered.

The influence of the state of stress on the strain accumulation is assumed to be independent from the material. As a consequence, the parameters $C_{\mathrm{f}}, C_{\mathrm{c}}, \eta_{\mathrm{c}}$, and $d_{0}$ are adopted from sand No. 3. Subsequently, the accumulation model parameters $\alpha_{\mathrm{f}}^{0}, \beta_{\mathrm{f}}^{0}, \theta_{\mathrm{f}}^{0}, \alpha_{\mathrm{c}}^{0}, \beta_{\mathrm{c}}^{0}$, and $\theta_{\mathrm{c}}^{0}$ are determined. 

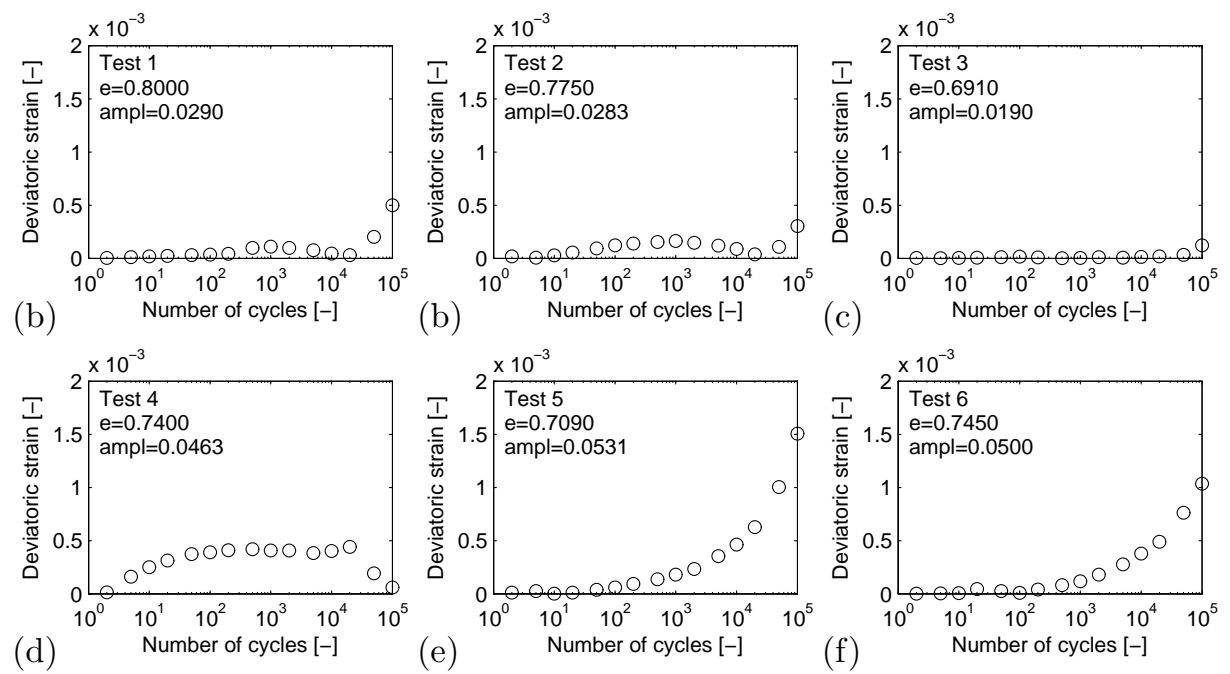

Figure 9: Measured accumulated deviatoric strain $\kappa^{\text {acc }}$ for tests LT1 to LT6.

Figure 9 shows the measured accumulated deviatoric strain. For tests LT1 to LT4 very small accumulated deviatoric strains $\kappa^{\text {acc }}$ are recorded as shown in Figure 9. These small values result in a relatively large measurement error. Therefore, the best fit for tests LT1 to LT4 is a vanishing $\kappa^{\text {acc }}$. This can be accounted for in the model by very large values of $\theta_{\mathrm{f}}^{0}$. In this case the influence of $\alpha_{\mathrm{f}}^{0}$ and $\beta_{\mathrm{f}}^{0}$ becomes small. They are thus set to default values. $\theta_{\mathrm{f}}^{0}$ is set to 100000 . With this high value, no significant changes in the resulting accumulation curves are detected.

Subsequently, a non-linear least squares procedure is used to fit equation (37) to the test data for the accumulated volumetric strain. This results in $\alpha_{\mathrm{c}}^{0}=16.3 \times 10^{-4}, \beta_{\mathrm{c}}^{0}=1.63 \times 10^{-6}, \theta_{\mathrm{c}}^{0}=86.68$, and $C_{\mathrm{e}}=85$. Figure 10 compares the experimental data with the respective calibrated accumulation curve. Since $\kappa^{\text {acc }}$ is small with respect to $\epsilon_{k k}^{\text {acc }}$, the influence of this error is minimized. An exception is the axial strain of test LT4 where the fitting error of $\kappa^{\text {acc }}$ becomes relevant. The accuracy of the prediction is satisfactory, despite the limited amount of test data. This is mainly related to the fact that the model parameters are insensitive to small changes of the void ratio and loading amplitude. A scatter of soil parameters of $\pm 10 \%$ in laboratory investigations or under in-situ conditions is not unusual. Accordingly, it is difficult to identify a causal connection of certain parameters. Furthermore, the character of the relation may vary for different soils.

\section{Conclusions}

In this paper, an accumulation model for settlements due to vibrations at small strain levels in granular non-cohesive soils has been proposed. It is assumed that the dynamic part of the loading is small with respect to the static part, reflecting the stress conditions in the soil underneath buildings. As the plastic deformation in the soil is only observed after a considerable amount of dynamic loading cycles, only the accumulation of the average plastic deformation is considered.

It is assumed that the accumulation is caused by two distinct mechanisms: frictional sliding and volumetric compaction of soil particles. Correspondingly, use is made of two state variables for frictional sliding and volumetric compaction, respectively. Following the observation that the direction of accumulation only depends on the stress state, the direction of accumulation is derived from the Mohr-Coulomb yield function.

Furthermore, the assumption of a common compaction curve has been made, implying that the shape of the compaction curve is invariant with respect to a variation of a parameter. Based on this assumption, a phenomenological law is put forward that describes the shape of the compaction curve. The strain accumulation rate is formulated independent from the number of load cycles and two independent accumulation 

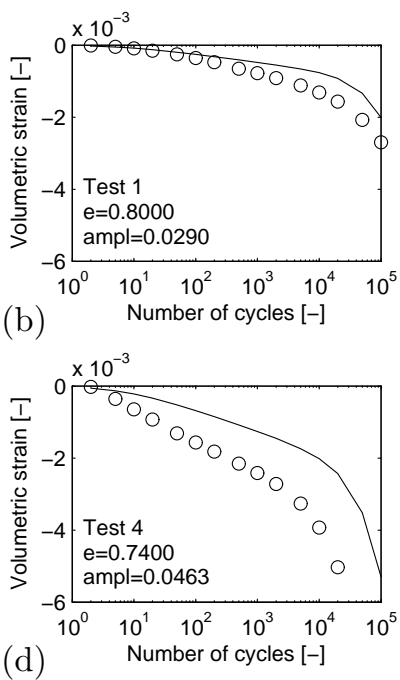

(d)

Number of cycles [-]

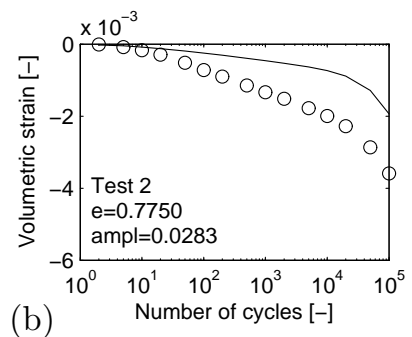

(b)

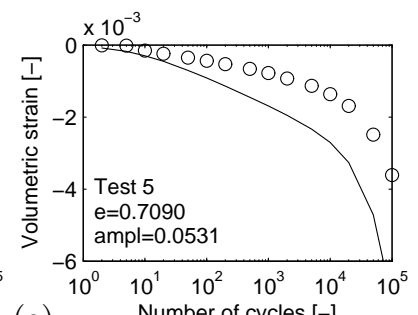

(e)

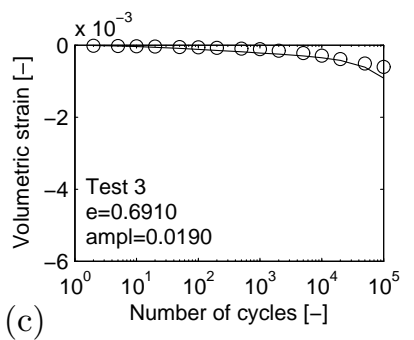

(c)

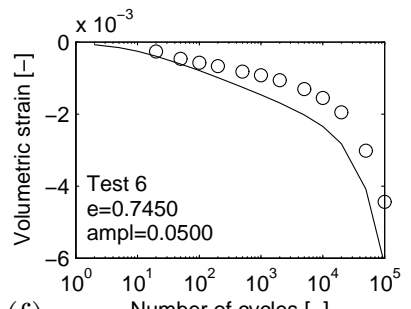

(f)

Figure 10: Measured and calibrated accumulated volumetric strains $\epsilon_{k k}^{\text {acc }}$ for tests LT1 to LT6.

laws for deviatoric and volumetric strain portions are introduced to ensure a flexible applicability of the model.

The presented accumulation model is calibrated to cyclic triaxial test results. Despite of several simplifications, coming along with the very limited number of model parameters, sufficient accuracy is reached in accumulation prediction. Although a broader data base is desirable, the prediction corresponds well to the test data.

\section{Acknowledgements}

The work presented in this paper is obtained within the frame of the SBO project IWT 03175 "Structural damage due to dynamic excitations: a multi-disciplinary approach". The financial support of the Flemish Community is gratefully acknowledged. The second author is a postdoctoral research fellows of the Research Foundation Flanders. The authors gratefully acknowledge financial support from these institutes. The supply of test data by Wichtmann [23], used for calibration of the new accumulation model, is highly appreciated.

\section{References}

[1] M. Abdelkrim, G. Bonnet, and P. de Buhan. A computational procedure for predicting the long term residual settlement of a platform induced by repeated traffic loading. Computers and Geotechnics, 30:463-476, 2003.

[2] F. Allou, C. Chazallon, and P. Hornych. A numerical model for flexible pavements rut depth evolution with time. International Journal for Numerical and Analytical Methods in Geomechanics, 31:1-22, 2006.

[3] M. Budhu. Soil Mechanics and Foundations. John Wiley \& Sons Inc., 2000.

[4] C.S Chang and R.V. Whitman. Drained permanent deformation of sand due to cyclic loading. Journal of Geotechnical Engineering, Proceedings of the ASCE, 114(10):1164-1180, 1988.

[5] S. Drabkin, H. Lacy, and D.S. Kim. Estimating settlement of sand caused by construction vibration. Journal of Geotechnical Engineering, Proceedings of the ASCE, 122:920-928, 1996.

[6] S. François, C. Karg, W. Haegeman, and G. Degrande. A numerical model for foundation settlements due to deformation accumulation in granular soils under repeated small amplitude dynamic loading. International Journal for Numerical and Analytical Methods in Geomechanics, 34(3):273-296, 2010.

[7] H.O. Fuchs and R.I. Stephens. Metal fatigue in engineering. Wiley, New York, 1980.

[8] T. Habiballah and C. Chazallon. An elastoplastic model based on the shakedown concept for flexible pavements unbound granular materials. International Journal for Numerical and Analytical Methods in Geomechanics, 29:577-596, 2005.

[9] C. Karg. Modelling of strain accumulation due to low level vibrations in granular soils. PhD thesis, Laboratory of Geotechnics, Ghent University, 2007. 
[10] J. Lemaitre and J.-L. Chaboche. Mechanics of solid materials. Cambridge University Press, Cambridge, 1990.

[11] M.P Luong. Mechanical aspects and thermal effects of cohesionless soils under cyclic and transient loading. In Proceedings of the IUTAM Conf. on Deformation and Failure of Granular Materials, pages 239-246, Delft, 1982.

[12] A. Niemunis, T. Wichtmann, and T. Triantafyllidis. Influence of a cyclic and dynamic loading history on dynamic properties of dry sand, part I: cyclic and dynamic torsional prestraining. Soil Dynamics and Earthquake Engineering, $24: 127-147,2004$.

[13] A. Niemunis, T. Wichtmann, and T. Triantafyllidis. Influence of a cyclic and dynamic loading history on dynamic properties of dry sand, part II: cyclic axial preloading. Soil Dynamics and Earthquake Engineering, 24:789-803, 2004.

[14] A. Niemunis, T. Wichtmann, and T. Triantafyllidis. A high-cycle accumulation model for sand. Computers and Geotechnics, 32:245-263, 2005.

[15] P. Perzyna. Fundamental problems in viscoplasticity. Record in Advanced Applied Mechanics, 9:367-395, 1966.

[16] S. Pycko and G. Maier. Shakedown theorems for some classes of nonassociative hardening elasticplastic material models. International Journal of Plasticity, 11:367-395, 1995.

[17] A. Sawicki and W. Swidzinski. Mechanics of a sandy subsoil subjected to cyclic loadings. International Journal for Numerical and Analytical Methods in Geomechanics, 12:511-529, 1989.

[18] A.S.J. Suiker. Fatigue behaviour of granular materials. Part 2: Numerical formulation of the constitutive relations. Technical report 7-98-119-3, Faculty of Civil engineering, Delft University of Technology, 1998.

[19] A.S.J. Suiker. The mechanical behaviour of ballasted railway tracks. PhD thesis, Delft University of Technology, 2002.

[20] A.S.J. Suiker and R. de Borst. A numerical model for the cyclic deterioration of railway tracks. International Journal for Numerical Methods in Engineering, 57(4):441-470, 2003.

[21] K. Thooft. Theoretisch experimentele beschrijving van het spannings vervormingsgedrag van onsamenhangende gronden onder uitwendige dynamische belastingen, in het bijzonder bij grote verglijdingen. PhD thesis, Department of Civil Engineering, Universiteit Gent, 1992.

[22] P.A. Vermeer. Formulation and analysis of sand deformation problems. PhD thesis, Delft University of Technology, Delft, The Netherlands, 1980.

[23] T. Wichtmann. Explicit accumulation model for non-cohesive soils under cyclic loading. PhD thesis, Ruhr Universität Bochum, Germany, 2005. 